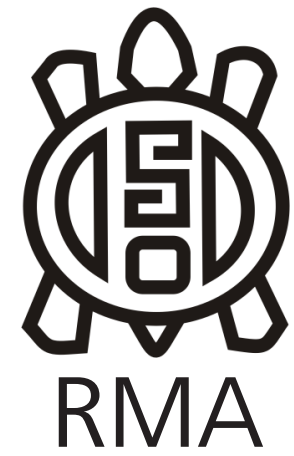

Dossier

\title{
Distribución de obsidianas en el NOA durante el Formativo: aportes desde las lascas de cuatro casos de estudio
}

\author{
Obsidian distribution in Northwestern Argentina during Formative \\ Period: contribution from flakes of four study cases \\ Cecilia Mercuri*
}

\begin{abstract}
${ }^{*} \mathrm{CONICET/ICSOH}$ (Consejo Nacional de Investigaciones Científicas y Técnicas/ Instituto de Investigaciones en Ciencias Sociales y Humanidades).

Email: ce_mercuri@yahoo.com.ar
\end{abstract}

\begin{abstract}
Resumen
Desde el proyecto de investigación Variabilidad Tecnológica y Redes de Interacción Social en el Noroeste Argentino a Través del Estudio de las Estrategias Tecnológicas Líticas durante el Período Formativo, se analizan conjuntos líticos de diversas áreas del Noroeste argentino en función de ampliar los estudios referidos a las esferas de distribución de obsidianas en torno a los 2000Ap. Los conjuntos provienen de: Puesto Viejo 2, en la provincia de Tucumán, Cueva de Cristóbal, en Jujuy, y SSalLaV 1 (8) y Salvatierra, ambos en la provincia de Salta. En este trabajo se presenta un análisis comparativo entre los conjuntos líticos mencionados, haciendo hincapié en la representación y tratamiento de obsidianas alóctonas, indagando en las diversas escalas y en la diversidad de fuentes utilizadas, teniendo en cuenta la oferta local de materias primas en el área de los sitios.
\end{abstract}

Palabras clave: material lítico; obsidianas alóctonas; NOA; redes de interacción social; Formativo

\begin{abstract}
From research project Technological variability and social interaction networks in Northwestern Argentina through the study of lithic technology y Formative Period, lithic assemblages from diverse areas in Northwestern Argentina are analyzed with the main goal of widening studies regarding obsidian distribution spheres at about $2000 \mathrm{Bp}$. Assemblages come from: Puesto Viejo 2, in Tucumán province, Cueva de Cristóbal, in Jujuy, and SSalLaV 1(8) and Salvatierra, both of them in Salta province. In this paper a comparative analysis among mentioned assemblages is presented. The spotlight is on representation and treatment dedicated to non local obsidians, looking for patterns in different scales and provenance sources linking raw material local offer in the sites area.
\end{abstract}

Keywords: lithic material; non local obsidians; Northwestern Argentina; social interaction networks; Formative Period

\section{Introducción}

Desde 2006 me encuentro trabajando en el análisis de material lítico proveniente de diversos conjuntos del Noroeste Argentino con fechados en torno a los 2000AP. En estos estudios se observó que los patrones que presentaban los conjuntos líticos no se explicaban en su totalidad por las pautas de movilidad y aspectos funcionales, sino que se destacaba la importancia de la organización social y el fortalecimiento de redes de interacción y circulación de obsidiana (Yacobaccio et al 2002). Actualmente, desde el proyecto de investigación Variabilidad Tecnológica y Redes de Interacción Social en el Noroeste Argentino a Traves del Estudio de las Estrategias Tecnologicas Liticas durante el Periodo Formativo, se analizan conjuntos líticos de diversas áreas del Noroeste argentino, los cuales fueron elegidos, en parte, en función de ampliar los estudios referidos a las esferas de distribución de obsidianas en otras zonas ecológicas más allá de la Puna. Los conjuntos provienen de: Puesto Viejo 2, Cueva de Cristóbal, SSalLaV 1 (8) y Salvatierra (figura 1).

Puesto Viejo 2, se ubica en Quebrada de Los Corrales, ubicada sobre el Abra de El Infiernillo a una altura promedio de 3100 msnm, en la provincia de Tucumán (Oliszewski et al 2008); Cueva de Cristóbal, en la localidad del Aguilar, en la Puna de Jujuy. Este sitio se encuentra a una altitud de unos $4.000 \mathrm{msnm}$ aproximadamente y ha sido investigado arqueológicamente por Jorge Fernández en la década de 1960 (retomados por Salomón Hocsman) (Fernández 1988-89). El sitio SSalLaV 1 (8), se localiza en el área del Cabra Corral, a 1100 msnm en Valle de Lerma, Salta, en un ambiente de transición entre parque 


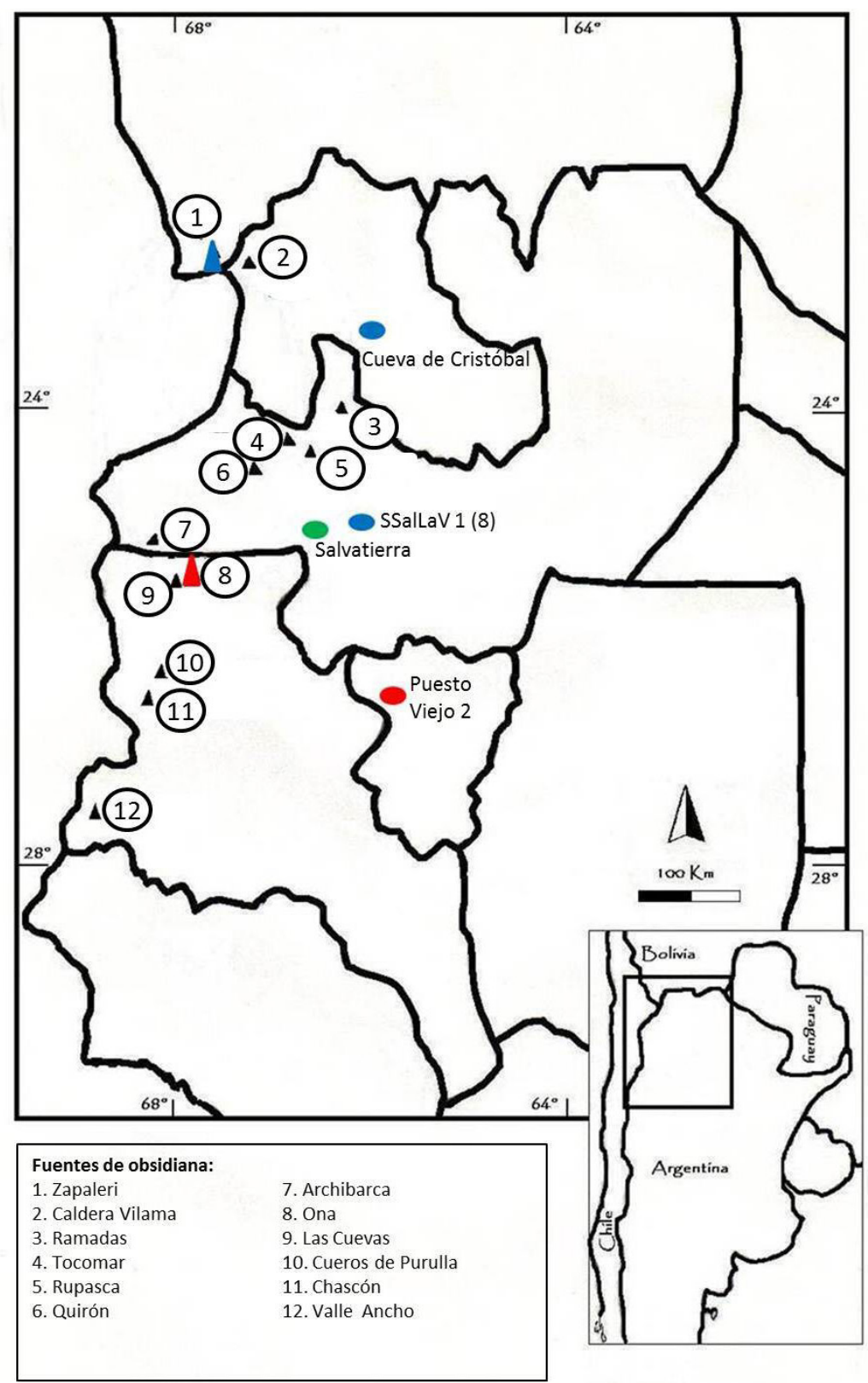

Figura 1. Esquema con los sitios mencionados en el texto y algunas fuentes de obsidiana.

Figure 1. Scheme with sites mentioned in the text and some obsidian sources.

chaqueño y desierto (Pantorrilla Rivas 2009). Asimismo, recientemente, se incorporó el análisis del material lítico de Salvatierra, en Cachi, provincia de Salta (Rivolta y Cabral Ortiz 2017). Las evidencias arqueológicas de este sitio comprenden un sector con ocupación sobre una antigua terraza fluvial nivelada que, en época de precipitaciones, se ve afectada por eventos de escurrimiento. Si bien todos los conjuntos líticos provienen de excavaciones estratigráficas también se incluyen datos de la presencia de materias primas en superficie.

Desde hace por lo menos dos mil años el paisaje de los Andes Meridionales cambia: empiezan a ocuparse espacios y aumentan las conexiones, relaciones y dependencias entre humanos y no humanos (Tarragó 1996). Procesos de sedentarización y aglomeración, domesticación de plantas y animales, innovaciones tecnológicas, crecimiento demográfico y nuevas negociaciones de poder político son características de estos momentos (Tarragó 1992). Asimismo, las cadenas de interdependencia se complejizan y probablemente se conforman nuevas temporalidades, lógicas y vínculos (Franco Salvi 2018). Sin embargo, a lo largo del primer milenio, las trayectorias históricas de cada sector del NOA fueron variando marcadamente $y$, a diferencia de los períodos anteriores y posteriores donde hay recurrencias 
macrorregionales mucho más intensas, estas sociedades transitaron recorridos muy particulares (Franco Salvi 2018).

Los objetos o las cosas producto de las tecnologías se encuentran entramados en las relaciones sociales, por lo que no deben ser considerados simples instrumentos prácticos (Scattolin y Lazzari 1997). Los individuos activamente producen y reproducen el mundo en el cual viven mediante herramientas y técnicas (Chaparro 2012). Diversas investigaciones en el Noroeste Argentino evidencian que desde el Formativo hay una tendencia a la informalidad de los conjuntos artefactuales sobre rocas locales (ej. Escola 2000), con excepción de las puntas de proyectil, las cuales están confeccionadas sobre obsidiana y presentan mayor inversión de trabajo (Lazzari 2005; Hocsman y Escola 2006-07). Estas materias primas, se encuentran localizadas en lugares particulares del paisaje, ya que es vidrio volcánico (Mercuri y Restifo 2014). No obstante, la diversidad de fuentes potenciales (Figura 1), la gran mayoría de los artefactos responden a dos variedades: Laguna Blanca/ Zapaleri se distribuye hacia el norte y Ona, hacia el sur (Yacobaccio et al 2002).

En este trabajo se presenta un análisis comparativo entre los conjuntos líticos de los sitios mencionados. Esto se realiza poniendo el foco en la representación y tratamiento de obsidianas alóctonas. Dado que cada conjunto se encuentra en distintas etapas de análisis, en este artículo se aborda el estudio de lascas de cada conjunto.

\section{Materiales y métodos}

El protocolo de análisis seguido se basó en la propuesta de
Aschero 1975 y 1983 y adecuaciones posteriores (Aschero y Hocsman 2004 y Mercuri 2011). Como ya se aclaró, los conjuntos provienen de excavación y de superficie y se consideraron únicamente las lascas. Las variables que se tomaron en cuenta para este análisis son el tipo de lasca (si son angulares, planas, etc.), el tamaño (hípermicrolascas, microlascas o lascas), el módulo de longitud/ anchura (las categorías propuestas por Aschero se englobaron en anchos, normales y alargados) y los talones (liso, puntiforme, diedro). Si bien en los estudios particulares se contemplan posibles diferencias entre distintos niveles de excavación, en este caso, la información se presenta de manera conjunta de modo de dar un panorama amplio.

La muestra de Puesto Viejo 2 se compone de un total de 251 piezas, principalmente de cuarzo y andesita, las cuales son materias primas locales (Cruz et. al. 2009, Mercuri y Mauri 2015). Las lascas enteras y fracturadas con talón, representan un $65 \%$ de la muestra $(n=163)$. El conjunto de Cueva de Cristóbal se compone de 681 piezas, de las cuales 386 son lascas enteras y fracturadas con talón. El conjunto presenta una gran diversidad de materias primas, tanto locales como alóctonas. Para SSalLaV 1 (8, en principio se contaba con 594 piezas. Una vez descartadas las indeterminadas y las fracturadas sin talón, la muestra quedó conformada por 344 especímenes. Las materias primas son principalmente areniscas locales recogidas en las márgenes de ríos tales La Viña, Ampascachi o Guachipas (Mercuri 2018). Por último, se analizaron 162 lascas provenientes de Salvatierra, donde, se registraron 8 variedades de materias primas locales, pero por el momento no se recuperaron materias primas alóctonas. Sin embargo, considero que este vacío es relevante para el análisis.

\begin{tabular}{|c|c|c|c|c|c|c|c|c|c|c|c|c|c|c|c|c|}
\hline \multirow[t]{4}{*}{ Sitio } & \multirow[t]{4}{*}{ locales } & \multicolumn{15}{|c|}{ No locales } \\
\hline & & \multirow[t]{3}{*}{ otras } & \multicolumn{14}{|c|}{ Obsidianas } \\
\hline & & & \multirow[t]{2}{*}{ variedad } & \multirow[t]{2}{*}{$\mathrm{n}$} & \multicolumn{3}{|c|}{ tipo de lasca } & \multicolumn{3}{|c|}{ tamaño } & \multicolumn{3}{|c|}{ módulo L/A } & \multicolumn{3}{|c|}{ talones } \\
\hline & & & & & $a$ & $p$ & i & $\mathrm{hm}$ & $\mathrm{m}$ & 1 & an & $\mathrm{n}$ & al & 1 & $p$ & d \\
\hline $\begin{array}{l}\text { Puesto } \\
\text { Viejo } 2\end{array}$ & 156 & 1 & Ona & 6 & 6 & - & - & 3 & 3 & - & 2 & 4 & - & 6 & & \\
\hline \multirow{3}{*}{$\begin{array}{l}\text { Cueva de } \\
\text { Cristóbal }\end{array}$} & \multirow[t]{3}{*}{296} & \multirow[t]{3}{*}{18} & Zapaleri & 45 & 38 & 5 & 2 & 44 & 1 & - & 11 & 26 & 8 & 25 & 20 & - \\
\hline & & & Tocomar & 26 & 23 & 3 & - & 26 & - & - & 16 & 9 & 1 & 19 & 7 & - \\
\hline & & & No dif. & 1 & 1 & - & - & 1 & - & - & - & - & 1 & - & 1 & - \\
\hline \multirow{2}{*}{$\begin{array}{l}\text { SSalLav } 1 \\
\text { (8) }\end{array}$} & \multirow[t]{2}{*}{315} & \multirow[t]{2}{*}{1} & Zapaleri & 27 & 21 & 2 & 4 & 17 & 5 & 5 & 17 & 10 & - & 20 & 3 & 4 \\
\hline & & & No dif. & 1 & 1 & - & - & - & - & 1 & - & 1 & - & 1 & - & - \\
\hline Salvatierra & 162 & & - & - & - & - & - & - & - & - & - & - & - & - & - & - \\
\hline
\end{tabular}

Tabla 1. Resultados relativos a las obsidianas no locales. Donde: tipo de lasca $a=$ angular, $p=p l a n a$ e $i=$ indeterminada; tamaño: $\mathrm{hm}=$ hípermicrolasca, $\mathrm{m}=$ microlasca $\mathrm{y} \mathrm{l}=$ lasca; módulo L/A: $\mathrm{an}=$ ancho, $\mathrm{n}=$ normal y al= alargado; talones: I=liso, $\mathrm{p}=$ puntiforme $\mathrm{y} \mathrm{d}=$ diedro.

Table 1. Results regarding non-local obsidians. Where: type of flake $a=$ angular, $p=$ flat and $i=$ undetermined; size: $h m=$ hypermicroflake, $m=$ microflake and I= flake; L/ A module: $a n=$ width, $n=$ normal and al=elongated; heels: I= smooth, $p=$ punctate and $d=$ dihedral 


\section{Resultados}

Como se observa en la tabla 1, en Puesto Viejo 2, si bien priman las materias locales (cuarzo y andesita) se registró la presencia de obsidiana proveniente de una única fuente, Ona (4\%). Éstas son lascas angulares tendientes a los tamaños pequeños (tabla 1). De anchos normales y anchos, todas las piezas poseen talones lisos. Por otra parte, en Cueva de Cristóbal, el conjunto presenta una gran diversidad de materias primas, tanto locales como alóctonas. Entre las primeras se registró variedad de calcitas, cuarcitas, areniscas, pizarra, cuarzo, ftanita y sílices. Entre las segundas, vulcanitas y 4 variedades de obsidiana macroscópicamente distintas (dos de la fuente de Zapaleri/Laguna Blanca, una de Tocomar y otra que no pudimos identificar, aunque se trate de un solo especimen). Las obsidianas representan el 19\% de esta muestra. Priman ampliamente las lascas angulares, seguidas en muy baja frecuencia por planas. Otros estados de este atributo no presentan valores significativos. Un $40 \%$ de muestra son hípermicrolascas muy pequeñas (cf. Mercuri 2011). En relación a las características litotéticas, en general predominan los módulos normales. Sin embargo, esta tendencia se revierte para la variedad Tocomar, donde son mayormente módulos de longitud/ anchura anchos. Con respecto a los talones, se observa una relativa alta frecuencia de talones puntiformes (20\%) en ambas variedades. En relación a las materias primas no locales de SSalLaV 1 (8) se registró una lasca pequeña en basalto, una en una variedad de obsidiana con muchas inclusiones y vesículas (No diferenciada) y 27 en obsidiana de la fuente de Zapaleri/ Laguna Blanca, alcanzando el $8 \%$ de la muestra. Se registra un predominio de lascas angulares. En relación a las características litométricas, si bien se trata principalmente de hípermicrolascas, a diferencia de los otros conjuntos, esta roca se presenta en tamaños mayores, siendo casi un 40\% microlascas y lascas (cf. Aschero 1983). Para las características litotéticas, la muestra tiende a los módulos anchos ( $F$ y G, cf. Aschero 1983). En este caso, también se observa una variación en relación a los otros conjuntos, ya que se registraron talones diedros además de los lisos. Tal vez esta característica responda al tamaño de los artefactos, aunque no pueden descartarse los modos de hacer (cf. Mercuri 2018).

Como ya se mencionó, el conjunto analizado de Salvatierra no registra materias primas alóctonas. Se compone de areniscas con diversa coloración y granulometrías provenientes principalmente de la Formación Cachi (Patricio Payola com pers.) y el porcentaje de corteza tiende a alto.

\section{Discusión y palabras finales}

Retomando, a partir del análisis de los diversos conjuntos se puede afirmar una cierta diversidad en relación a la presencia/ ausencia de obsidianas no locales. Mientras que en Cueva de Cristóbal se observa una relativa diversidad de estas rocas y en SSalLaV 1 (8) se evidenciaron 2 variedades y en Puesto Viejo2, tan solo una, en Salvatierra no se registra. Entonces, en este sentido, se detecta evidencia de las esferas de distribución de obsidianas (Yacobaccio et al 2002) en casi todos los contextos, siendo Zapaleri/ Laguna Blanca al norte y Ona al sur.

La frecuencia es asimismo muy variable. Mientras que en Cueva de Cristóbal es abundante la presencia de hípermicrolascas, en SSalLaV 1 (8) no se registra en abundancia pero sí en tamaños mayores. Las características de las lascas analizadas dan cuenta de por un lado, distintas etapas en las cadenas operativas, y por otro posibles funcionalidades diferentes de los sitios bajo estudio. Vale aclarar que considerando que este trabajo es una primera aproximación, la funcionalidad referida es meramente tentativa y en esta instancia generadora de hipótesis. En el primer sentido, se observa que el conjunto de Cueva de Cristóbal, probablemente al igual que el de Puesto Viejo 2, presenta las últimas etapas de formatización y regularización de filos de los artefactos y en SSalLaV 1(8), etapas intermedias. En el segundo sentido, hay que considerar que los contextos hayan tenido distintas funcionalidades. La ubicación de la Cueva de Cristóbal, un alero, la hace óptima para el avistamiento de fauna, para habitación de carácter no permanente (mas sí persistente), mientras que tanto Puesto Viejo 2 como SSalLav 1 (8) son unidades residenciales de carácter probablemente más doméstico. Así, aun considerando la variabilidad de estrategias de subsistencia sugeridas por la evidencia, se rescata que la presencia de obsidianas se corresponde a grandes rasgos con las esferas de interacción propuestas por Yacobaccio y colaboradores (2002). En este sentido, la evidencia paracada caso, muestra los distintos rangos de acción de esas poblaciones. Es decir, las áreas que abarca cada conjunto varían en distancia si nos remitimos a las obsidianas (figura 1). En el caso de Cueva de Cristóbal se registra que las obsidianas provienen de unos 100 Km (fuente Zapaleri/ Laguna Blanca), en SSalLaV 1(8) las obsidianas dan cuenta de distancias de unos 300 $\mathrm{Km}$ lineales a la fuente y en Puesto Viejo 2 la fuente de obsidiana Ona se localiza a más de $200 \mathrm{Km}$ del sitio. Si esto se combina con la abundancia en la cual aparecen, se observa una relativa mayor dependencia de obsidianas no locales en el caso de Cueva de Cristóbal que los otros, en los cuales aparecen moderadamente. Ahora bien, a diferencia de este conjunto, en el de SSalLaV 1 (8) se registran no ya las últimas etapas de formatización de artefactos sino las intermedias, evidenciando probables divergencias en el carácter de esta la transacción.

En todos los casos estudiados la disponibilidad de rocas locales es próxima e inmediata. Y en el conjunto de Salvatierra no se recuperaron obsidianas de ninguna 
clase. Esto habilita a preguntarnos acerca de las tomas de decisiones en torno a la obtención de materias primas líticas exógenas habiendo disponibilidad de rocas de calidad en el entorno. Es indudable que las elecciones no pasan únicamente por cuestiones funcionales o económicas y existen otros factores vinculados a particularidades de cada comunidad/ sociedad que podrían estar incidiendo (ver sobre cuestiones relacionadas Colombo y Flegenheimer 2013 y supra). En este sentido, alrededor del 2000 Ap se estaría desarrollando un proceso extremadamente complejo que va a originar cierto establecimiento de determinadas interacciones y estrategias sociales que involucrarán las diversas variedades de obsidianas y persistencia.

Salta, 28 de Marzo de 2019

\section{Agradecimientos}

A les coordinadores del Simposio en el cual se presentó una versión anterior de este trabajo, por la invitación a participar y la buena onda durante las presentaciones. A les compañeres que durante el mencionado Simposio hicieron aportes a esta contribución. Al geólogo Dr. Patricio Payola de la UNSa por ayudarme con la determinación de rocas. A Alfredo Calisaya por sus comentarios. A les evaluadores anónimes por sus valiosos aportes. No obstante, todo lo aquí vertido es de mi entera responsabilidad.

\section{Referencias citadas}

Aschero, C. A. 1975. Ensayo para una clasificación morfológica de artefactos líticos aplicada a estudios tipológicos comparativos. Informe presentado a CONICET. ms.

Aschero, C. A. y S. Hocsman 2004. Revisando cuestiones tipológicas en torno a la clasificación de artefactos bifaciales. Temas de arqueología. Análisis lítico. Compilado por A. Acosta, D. Loponte y M. Ramos, (pp. 7-25). Luján. Universidad Nacional de Luján.

Chaparro, M. G. 2012. La Tecnología Lítica como Fenómeno Multidimensional. El caso de las Sociedades Preestatales y Estatales del Valle Calchaquí Medio. Relaciones de la Sociedad Argentina de Antropología XXXVII: 355- 386.

Colombo, M. y N. Flegenheimer 2013. La elección de rocas de colores por los Pobladores Tempranos de la Región Pampeana (Buenos Aires, Argentina). Nuevas Consideraciones desde las Canteras. Boletín del Museo Chileno de Arte PrecolombinoVol. 18, № 1: 125-137.

Cruz H., Mauri E.P. y Martínez J.G. 2009.Reconocimiento de fuentes de aprovisionamiento prehispánicas de materias primas líticas en la Quebrada de los Corrales (El Infiernillo, Tucumán. Argentina). Serie Monográfica Didáctica Vol. 48. Facultad de Ciencias Naturales e IML, Tucumán. Argentina, pág. 111.

Escola, P. S. 2000. Tecnología Lítica y sociedades agropastoriles tempranas. Tesis Doctoral. Buenos Aires, Facultad de Filosofía y Letras. Universidad Nacional de Buenos Aires.

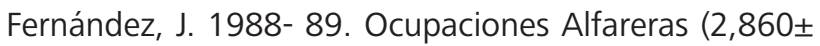
160 años AP) en la Cueva de Cristóbal, Puna de Jujuy, Argentina. Relaciones de la Sociedad Argentina de Antropología Tomo XVII/ 2 N. S.: 139- 182.

Franco Salvi, V. 2018. Autonomía doméstica en un mundo complejo (valle de Tafí, Argentina). Boletín de Arqueología PUCP N. ${ }^{\circ}$ 24: 55-76.

Hocsman, S. y P. Escola. 2006-2007. Inversión de trabajo y diseño en contextos líticos agropastoriles (Antofagasta de la Sierra, Catamarca). Cuadernos del Instituto Nacional de Antropología y Pensamiento Latinoamericano 21: 75-90.

Lazzari, M. 2005. The Texture of Things: Objects, People, and Landscape in Northwest Argentina (First Millennium A.D.). En: Archaeology of Materiality Editado por L. Meskell, (pp. 126-161). Oxford. Blackwell.

Mercuri, C. 2011.De rocas, pastores y agricultores. La tecnología lítica de los primeros productores de alimentos de la puna de Salta, Argentina. Editorial Académica Española.

Mercuri, C. 2018. Experimental flintknnaping with sandstones from Cabra Corral area, Salta, Argentina. Journal of Lithic Studies 5 (2). En prensa.

Mercuri, C. y E. Mauri 2015. La ocupación humana en la Quebrada de Los Corrales (Tucumán, Argentina) hacia el $1^{\circ}$ milenio de la era Cristiana: análisis del material lítico de PV2 Estructura 1. Cuadernos del Instituto Nacional de Antropología y Pensamiento Latinoamericano - Series Especiales 2 (1):122-128.

Mercuri, C. y F. Restifo 2014. Application of physicochemical and macroscopic methods to lithic artifact studies from Alero Cuevas site (Salta, República Argentina): a complementary approach. En Physical, chemical and biological markers in argentine archaeology: theory, methods and applications. Editado por D. M. Kligmann y M. R. Morales. BAR International Series 2678. Archaeopress, Oxford.

Oliszewski, N., J. Martínez y M. Caria 2008. Ocupaciones prehispánicas en una quebrada de altura: el caso de Cueva de los Corrales 1 (El Infiernillo, Tafí del Valle, 
Tucumán). Relaciones de la Sociedad Argentina de Antropología XXXIII: 209-221.

Pantorrilla Rivas, M. M. 2009. Sitio Vaquerías en Cabra Corral, Departamento de La Viña Provincia de Salta, Argentina. Trabajo presentado en Quinto Congreso de Estudios Bolivianos, Sucre 24 a 27 de Junio de 2009

Rivolta, M. C y J. E Cabral Ortiz 2017. El espacio doméstico en las ocupaciones aldeanas del valle Calchaquí Norte (Salta, Argentina). Arqueología Iberoamericana 36: 66-78.

Scattolin, M. C. y M. Lazzari 1997. Tramando redes: Obsidianas al oeste del Aconquija. Estudios Atacameños 14:189- 209.
Tarragó, M. N. 1992. El Formativo y el Surgimiento de la Complejidad Social en el Noroeste Argentino. En Formativo Sudamericano, Una Reevaluación. Homenaje a A.R. González y B.J. Meggers Editado por P. LedergerberCrespo, (pp. 302- 313). Quito. Abya- Yala.

Tarragó, M. 1996. El Formativo en el Noroeste Argentino y el alto valle Calchaquí, Actas y Memorias del XI Congreso Nacional de Arqueología Argentina, Revista del Museo de Historia de San Rafael 23, 103-119, Mendoza.

Yacobaccio, H, P. Escola, M. Lazzari y F. Pereyra. 2002. Long-Distance obsidian Traffic in northwestern Argentina. En Geochemical evidence for Long-Distance Exchange. Editado por M. Glascock.Scientific archaeology for the Third Millennium. Bergin and Garvey, Wesport. 